Volume 19, No 3 International Journal of Radiation Research, July 2021

\title{
Evaluation of volumetric modulated arc therapy technique for cranio-spinal irradiation: A rando - phantom based dosimetric study
}

\author{
E.O. Goksel ${ }^{*}$, E. Tezcanli², M. Okutan ${ }^{3}$, H. Bilge3, Y. Yildiz Yarar4 \\ ${ }^{1}$ Acibadem Mehmet Ali Aydinlar University, Vocational School of Health Services, Radiotherapy Program, Istanbul, \\ Turkey \\ ${ }^{2}$ Acibadem Altunizade Hospital, Department of Radiation Oncology, Istanbul, Turkey \\ ${ }^{3}$ Istanbul University Medicine Faculty, Oncology Institute, Department of Radiotherapy, Istanbul, Turkey \\ ${ }^{4}$ Yildiz Technical University, Department of Physics, Istanbul, Turkey
}

\section{- Original article}

\section{*Corresponding authors:}

Evren O. Goksel, Ph.D.,

E-mail:

e.ozangoksel@gmail.com

Revised: May 2020

Accepted: June 2020

Int. J. Radiat. Res., July 2021; 19(3): 531-541

DOI: $10.29252 /$ ijrr.19.2.531

\begin{abstract}
Background: In this study, clinical advantages and the dosimetric accuracy of volumetric modulated arc therapy (VMAT) technique for cranio-spinal irradiation (CSI) were investigated and compared with field in field (FinF) and intensity modulated radiotherapy (IMRT) techniques. Materials and Methods: The organs at risk (OARs) and planning target volume (PTV) were generated on the RANDO phantom computerized tomography (CT) image series. The dose-volume parameters for PTVs and OARs were compared for three techniques. Furthermore, the dose distribution inside the RANDO phantom was measured with thermoluminescent dosimeter (TLD) for all three techniques. Comparisons were done between measured and calculated doses of field junctions, targets and OARs. Results: FinF failed to obtain an adequate dose distribution for the upper spine (US), while VMAT and IMRT provided good dose coverage for all parts of the PTV. The most homogeneous dose distribution at the field junctions was obtained with the VMAT. Although heart, thyroid, intestine, lung, liver and testicular maximum doses were lower for the VMAT, the mean doses were higher except for the heart and thyroid. The absolute volume receiving prescription dose was significantly lower for the VMAT. The median differences between the TLD measurements and TPS calculations were $0.27 \%(7.8,-9.7 ; p=0.394),-0.28 \%(8.1,-10.7 ; p=0.322)$ and $0.33 \%(8.4,-12 ; p=0.338)$ for the FinF, IMRT and VMAT, respectively. These differences were not found statistically significant for the VMAT technique, as were the other two techniques. Conclusion: We conclude that the VMAT technique is the optimal Linac based CSI treatment in regards to dose coverage, dose homogeneity in field junctions and OAR sparing for higher doses. In addition, the VMAT showed dose distribution accuracy as good as the two other techniques in measurements simulating real clinical situations.
\end{abstract}

Keywords: Cranio-spinal irradiation, VMAT, TLD, RANDO phantom.

\section{INTRODUCTION}

Cranio-spinal irradiation (CSI) is one of the most challenging techniques in radiation therapy. With two laterally opposed cranial fields, matched to a posterior spinal field, this technique results in a dose inhomogeneity, especially at the junctions, and a significant dose anterior to the spinal target volume. There are some risks associated with CSI, such as radiation -induced myelitis in the case of overdosage and local failure if there is under dosage along the field-matching region. Delivering the prescribed dose to the target volumes is essential for tumor 
control, and protecting the normal tissue is important to reduce toxicity and risk of developing secondary cancers (SC) (1-3).

Nowadays, pencil scanning beam proton therapy is considered to be the most convenient treatment option for CSI because of the special depth dose characteristics $(4,5)$. On the other hand, scanning beams (protons) are not available in many countries (6). For this reason, conventional field-in-field (FinF), Intensity Modulated Radiotherapy (IMRT) and Volumetric Modulated Arc Therapy (VMAT) techniques with high-energy photons are used in the treatment of CSI in most of the world.

The clinical worldwide use of the VMAT technique has been significantly increasing, given the improved conformal dose distribution within the target, better normal tissue sparing and more homogeneous dose distribution in the field junctions (7). It provides shorter treatment delivery time and reduction in monitor unit (MU) usage when compared to fixed gantry IMRT (8). There are feasibility studies investigating the clinical suitability of VMAT technique and comparing it to other techniques based on the evaluation of dose distributions and dose-volume histograms (DVH) on the treatment planning system (TPS) for different clinical cases including CSI $(9,10)$. Fogliata etal. reported five different institutes' VMAT CSI treatment results for five patients (11). The results indicated that this technique was applicable to be used in clinical practice and could be advantageous in treating patients requiring CSI. The planar dose check for plan verifications was evaluated; however dosimetric measurements simulating the real treatment conditions were not reported in this study. But dosimetric accuracy should be tested before implementing a new technique as complicated as VMAT-CSI for patient treatment and the precision of the TPS should be confirmed.

There are several studies in the literature evaluating the dosimetric accuracy of conventional techniques for CSI, such as those of Baghani et al., but there is no comprehensive dosimetric study for VMAT-CSI (12-15). Baghani et al. investigated the dosimetric accuracy of CorePlan 3-dimensional (3D) TPS for conformal
CSI technique using RANDO phantom and GR-200 thermoluminescent dosimeter (TLD). They concluded that measured dose results support the accuracy of the treatment planning software.

Hood et al. investigated correlation of 3D-planned and measured dosimetry of photon and electron craniospinal radiation in a pediatric anthropomorphic phantom and they concluded that the 3D TPS predicted dose distribution matches the delivered dose in most instances (13). Besides Shoa et al. measured lens doses with TLD in 15 pediatric patients for 3D conformal radiotherapy (3DCRT) technique and showed the difference with planning as $6.5 \%{ }^{(14)}$.

Panandiker etal. reported a new technique that aimed to improve dose coverage and obtain a more homogeneous dose distribution for CSI with IMRT (15). Twelve patients' computerized tomography (CT) image series were used for CSI planning with both conventional and IMRT techniques. The plans were compared for dose homogeneity in the target, and treatments were applied with the IMRT technique. Film dosimetry was used to verify the field-matching dose between cranial and spinal fields.

In this study, the clinical advantages and dosimetric accuracy of the VMAT technique for CSI on a RANDO phantom were investigated and compared with those of the conventional FinF and IMRT techniques. To our knowledge, this is the first study to perform extensive dosimetric measurements in a humanlike phantom for the VMAT-CSI technique

\section{MATERIALS AND METHODS}

\section{Contouring}

The humanlike RANDO phantom (CIRS ATOM ${ }^{\circledR}$ Dosimetry Phantoms, Model 701, CIRS Inc., VA) was placed in a supine position without using any immobilization devices, and CT images with $3 \mathrm{~mm}$ slice thickness were obtained using a Picker PQ 5000 (Philips Medical Systems, Cleveland, OH). Although RANDO phantom does not provide the same complexity and variability as human anatomy, it enables us to perform both planning comparisons and delivery verification 
which are essential to developing a better understanding of limitations and pitfalls of this complex treatment technique.

To use the optimization process for IMRT and VMAT, PTV brain (whole brain plus $0.5 \mathrm{~cm}$ ) and PTV spinal (whole spinal canal plus $1 \mathrm{~cm}$ ) volumes were generated. RANDO phantom provided only bony anatomy, brain, lungs and spinal canal as structures. Therefore, in order to contour other OAR volumes (eyes, optic nerves, oral cavity, and heart, liver, thyroid, kidneys and small bowel), CT images of a previously treated patient whose physical anatomy was compatible with the phantom were registered to the phantom CT images and used as a reference for volume delineation. Non-target tissue was generated by subtracting target structures from the whole body.

\section{Treatment planning}

The total prescription dose was 36Gy in 1.8Gy/frx to the craniospinal axis. Varian Eclipse (version 8.6, Eclipse: Varian Medical Systems, Palo Alto, CA) TPS was used to create plans for VMAT, sliding window IMRT and FinF techniques. For VMAT plans, Progressive Resolution Optimizer (PROII) was used as an inverse planning optimizer and Anisotropic Analytical Algorithm (AAA) was used for dose calculations with a dose grid size set to $2.5 \mathrm{~mm}$. The dose-volume parameters for target volumes and OARs were compared for three treatment techniques.

\section{Conventional (FinF) technique}

For the FinF technique, the cranial part of the target volume was planned with two 6MV lateral opposing fields, and segmental corrections were done for overdoses at the frontal and occipital areas as described in Kusters et al. study (16). The inferior border of the cranial fields was matched to the superior border of the spinal field, with preference at the level of vertebrae C5/C6 to spare the mandible and avoid the shoulders as much as possible. A collimator rotation for the cranial fields was applied to match the divergence of the posterior spinal field. Since the RANDO phantom is an adult male, the length of it is not suitable to treat the spinal region with only one posterior field. For this reason, two posterior spinal segmented fields ( 6 and $18 \mathrm{MV}$ ) were generated. The couch was rotated $90^{\circ}$ for the lower spinal (LS) field, and the gantry angle was matched to the divergence of the inferior edge of the upper spinal (US) field.

\section{IMRT technique}

The same cranial fields that had already been generated with the FinF technique were used. The spinal region was divided into two components, and each component was separately planned using an inverse planning sliding window IMRT technique with 6MV five isocentric posterior oblique fields $(140,160$, 180,200 and 220 gantry angles) $(9,16,17)$. The US fields overlapped by $2 \mathrm{~cm}$ with the cranial and LS fields in order to provide a homogeneous dose distribution since these overlapping fields were accounted for by the optimization algorithm to reduce the dose gradient in the junctions. When optimizing the US plan, the cranial plan was used as a base plan. In the same way, when optimizing the LS plan, the US plan was used as a base plan.

\section{VMAT technique}

Three partial arcs for the cranial region and one full arc for each of the US and LS regions were used (figure 1). In the cranial arcs, 50-310 degree gantry angles were left empty in order to protect the lenses, and because of the arms, 245 -305 and 55-105 degree gantry angles were left blank in the spinal arcs. The upper spinal arc was overlapped $2 \mathrm{~cm}$ with both the cranial and LS arcs (18). All arcs were optimized in the same plan. Using a base dose plan and optimizing each arc individually for each isocenter did not provide sufficient dose homogeneity in the field junctions, therefore all arcs were optimized in the same plan.

Considering the clinical suitability of the plans, dose distributions of the axial-sagittal slices in terms of dose coverage in the target structures, dose homogeneity in the field junctions and the DVHs for the OARs doses were evaluated. 


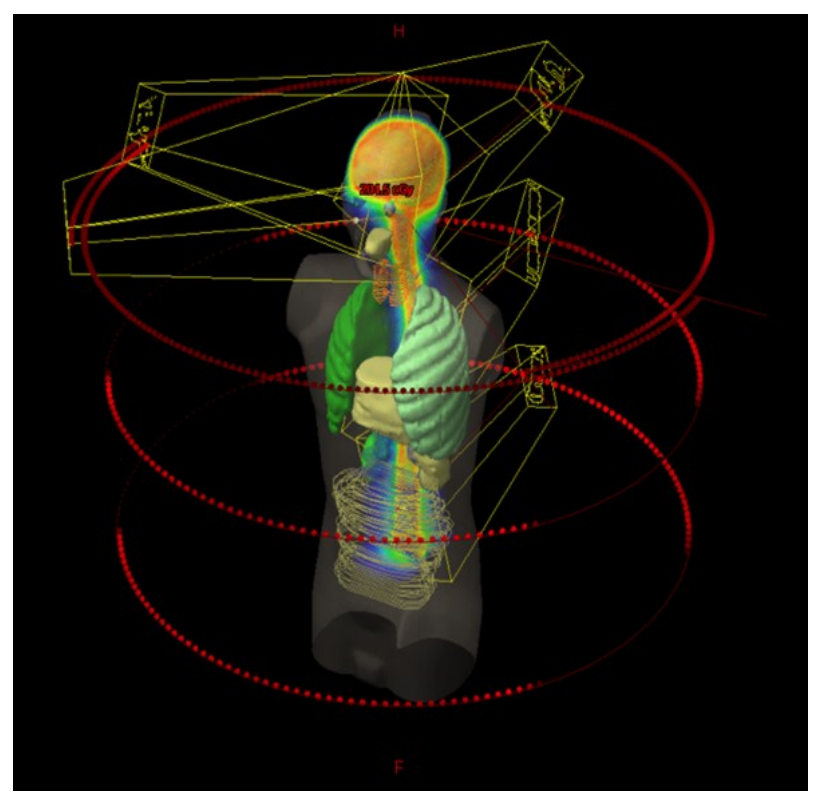

Figure 1. 3D arc arrangement for the VMAT technique. Three partial arcs for the cranial region and one full arc for each of the US and LS regions were used. There was no beam entry from the lenses in the cranial region and from the arms in the spinal areas.

\section{TLD calibration}

A total of 80 new GR-200A TLDs were used for this study. Before measurements, the individual TLD sensitivities were estimated by exposing them five times to a uniform dose of 1Gy from a Co60 beam under full scatter conditions. During these measurements, the field size was $20 \times 20 \mathrm{~cm}$, the source to skin distance was $80 \mathrm{~cm}$ and the depth of the TLDs was $5 \mathrm{~cm}$ in the solid water phantom. The TLD readings were performed with a Fimel (FIMEL, France) TLD reader. For each irradiation, the reading of each TLD within the group was compared to the average group reading to estimate the individual calibration factors. The standard deviations of the calibration factors were used to estimate individual TLD sensitivities. The 51 TLDs, which had a calibration factor within the range of $2 \%$ standard deviation, were selected to be used for point dose measurements in the humanlike phantom. The mean value of the individual standard deviation of each TLD chip was $1.6 \%$ $(0.8,2.4)$. Three of the most stable TLDs were kept as the calibration group and exposed to a known dose of $1 \mathrm{~Gy}$ at the calibration conditions immediately after the CSI delivery to the humanlike phantom.

\section{RANDO phantom irradiation}

Locations in the RANDO phantom for point dose measurements were determined using axial, coronal and sagittal plane views of the CT images on the TPS. The taps were pulled out from the RANDO phantom and were cut in the middle of the long axis and shortened from both sides to match the thickness of the TLD chips. The TLD chips were made into a sandwich between tap pieces and inserted into the same hole from which they were pulled out. The locations of the 23 TLDs inside the RANDO phantom are shown in figure 2 .

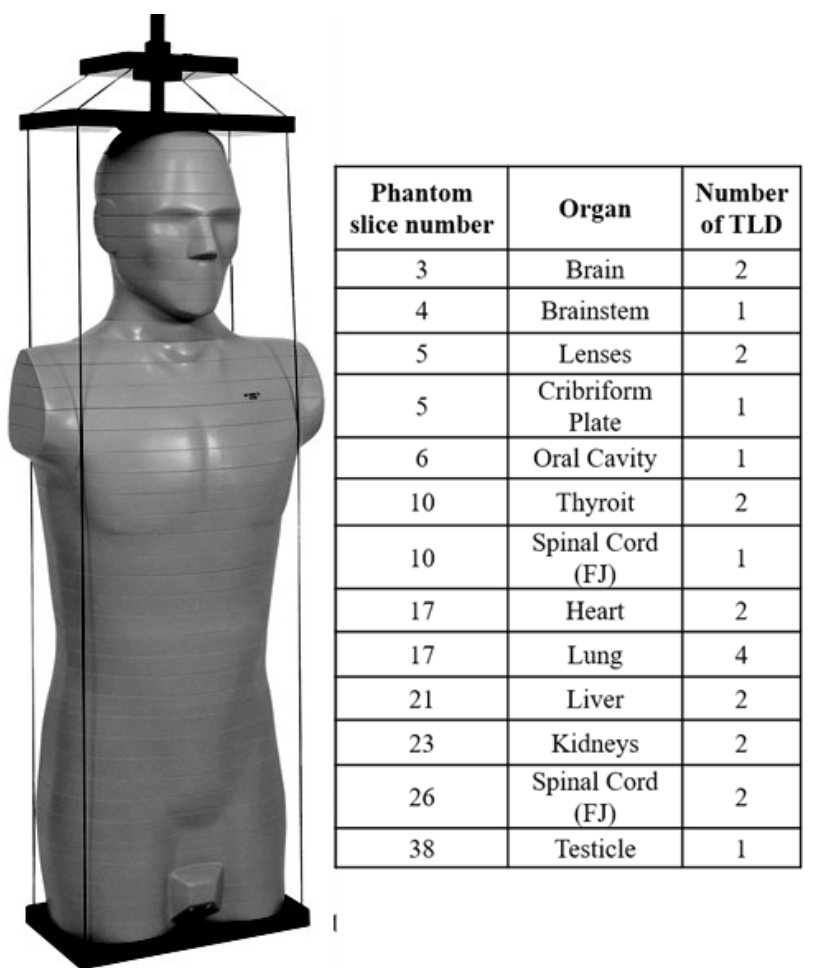

Figure 2. TLD locations and the number of TLD used are sorted by the slice number of the RANDO phantom in order to measure the delivered dose for each technique. FJ refers to Field Junction.

The RANDO phantom was irradiated with Varian Linac (Trilogy, Varian Medical Systems, and Palo Alto, CA) equipped with RapidArc and OBI with integrated Millennium 120MLC. Setup verifications were done using a $\mathrm{kV}-\mathrm{kV}$ match for all isocenters of all plans. Treatment plans generated for each technique were applied to the 
RANDO phantom 5 times in order to get 5 different readings from TLD measurements. The mean values for these 5 measurements were calculated. The calculated point doses where the TLDs were placed in the RANDO phantom were determined from the TPS and compared with the TLD readings. Thus, calculated versus measured dose differences were obtained.

\section{Statistical analysis}

The Wilcoxon test (Nonparametric version of paired-samples $t$-test) was used to evaluate differences between measured and calculated values. All hypotheses tested using a criterion level of $p=0.05$. Statistical analysis was performed by SPSS (IBM, Chicago, IL, USA) software version 23.0.

\section{RESULTS}

The comparative dose distributions in the sagittal views are presented for all techniques in figure 3 and figure 4 with an absolute dose color wash for (figure 3) 30Gy and (figure 4) 10Gy. The VMAT-CSI shows the most homogeneous dose distribution, especially in the field junction regions. The VMAT and IMRT demonstrate substantial dose reduction for the 10 Gy dose bath in the anterior area of the spine.

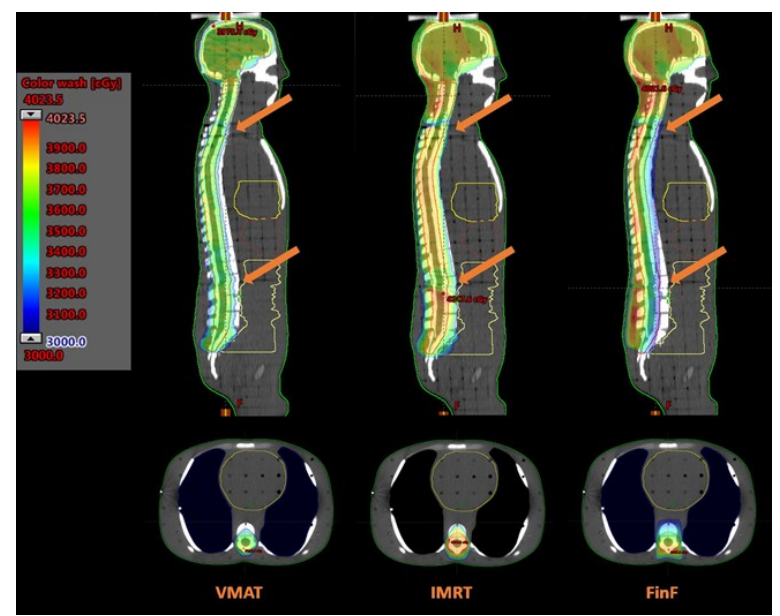

Figure 3. Sagittal and thoracal axial view, dose color wash comparison of VMAT, IMRT and FinF techniques for $30 \mathrm{~Gy}$. It is observed that the dose distribution in the field intersection regions is more homogeneous in the VMAT technique than the other two techniques (The orange arrows show the field intersection areas).

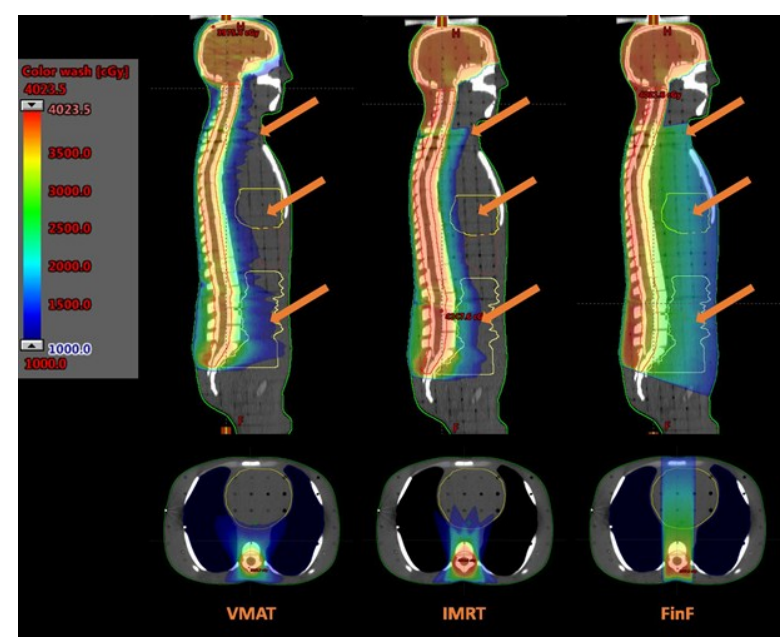

Figure 4. Sagittal and thoracal axial view, dose color wash comparison of VMAT, IMRT and FinF techniques for $10 \mathrm{~Gy}$. In particular, organs located in the anterior of the spinal region were exposed to a dose of $10 \mathrm{~Gy}$ in the FinF technique, while the IMRT and VMAT techniques better limited this dose (Orange arrows show these OARs located in the anterior of the spinal region).

\section{Target coverage}

The mean target doses and D95, D98, D2 and V107 values are shown in table1 to evaluate target coverage and hot spots. Although, VMAT and IMRT techniques achieved successful target coverage; Finf provided inferior target coverage, especially in the spinal region. When D95 doses were compared to PTV Spinal by the percentage of the prescribed dose, VMAT and IMRT provided more than $95 \%$, while FinF was able to provide only $88 \%$. The PTV Brain mean, D95 and D2 doses were (37.5, 36.2 and 38.8Gy), (38.4, 37.2 and 39.5Gy), (35.9, 34.5 and 37.4Gy) for the VMAT, IMRT and FinF techniques, respectively.

Table 1. Dose statistics of target structures according to the techniques used.

\begin{tabular}{|c|c|c|c|c|c|c|}
\hline & \multicolumn{2}{|c|}{ VMAT } & \multicolumn{2}{c|}{ IMRT } & \multicolumn{2}{c|}{ FinF } \\
\hline Target & $\begin{array}{c}\text { PTV } \\
\text { Brain }\end{array}$ & $\begin{array}{c}\text { PTV } \\
\text { Spinal }\end{array}$ & $\begin{array}{c}\text { PTV } \\
\text { Brain }\end{array}$ & $\begin{array}{c}\text { PTV } \\
\text { Spinal }\end{array}$ & $\begin{array}{c}\text { PTV } \\
\text { Brain }\end{array}$ & $\begin{array}{c}\text { PTV } \\
\text { Spinal }\end{array}$ \\
\hline Mean Dose (Gy) & 37.5 & 36.4 & 38.4 & 38.8 & 35.9 & 31.9 \\
\hline$D_{95 \%}(G y)$ & 36.2 & 34.6 & 37.2 & 36.9 & 34.5 & 31.9 \\
\hline$D_{98 \%}(G y)$ & 35.6 & 33.9 & 36.6 & 35.5 & 34.0 & 31.3 \\
\hline$D_{2 \%}(G y)$ & 38.8 & 38.0 & 39.5 & 40.0 & 37.4 & 39.2 \\
\hline$V_{107 \%}(\%)$ & 6.2 & 0.4 & 47.0 & 75.0 & 0.0 & 6.7 \\
\hline
\end{tabular}

When the volumes exposed to a high dose of $107 \%$ were evaluated within the target volumes, IMRT technique was very high with $47 \%$ for PTV brain and $75 \%$ for PTV spinal. Figures 5 and 6 
show the comparison of maximum and mean OAR doses for each technique in the bar chart. The lowest max doses were obtained with the VMAT technique for the liver, heart, thyroid, eyes, and lungs, IMRT technique for the kidneys and FinF technique for testicles.

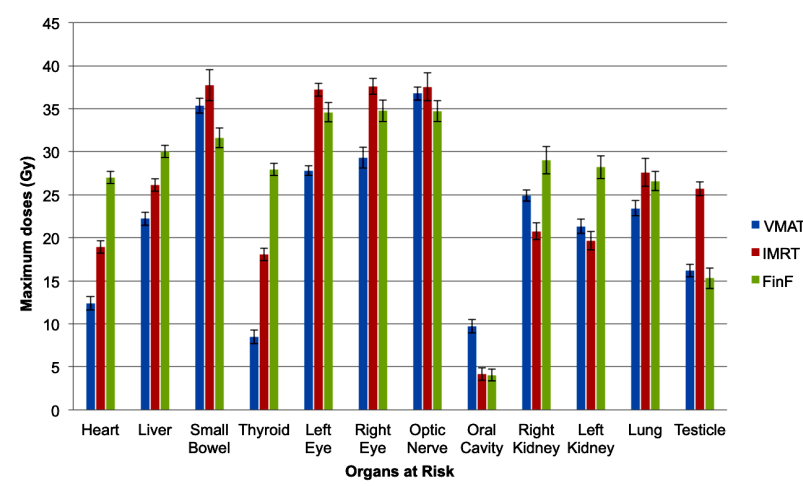

Figure 5. Organs at risk maximum doses were shown comparatively for VMAT, IMRT and FinF techniques.

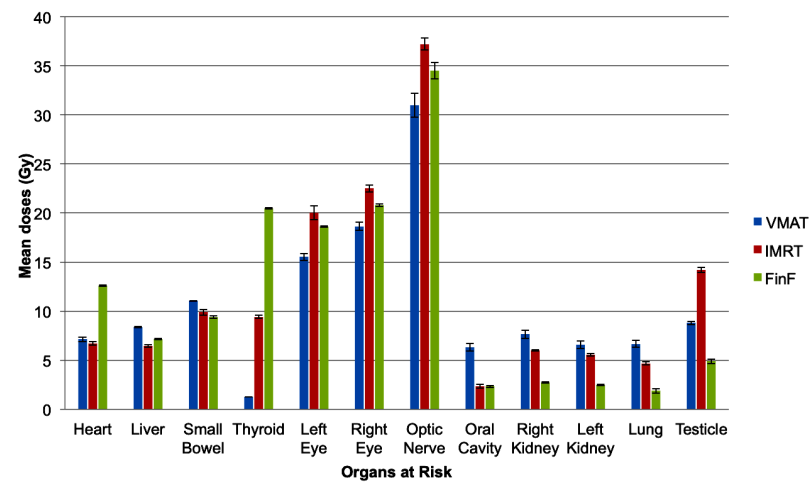

Figure 6. Organs at risk mean doses were shown comparatively for VMAT, IMRT and FinF techniques.

Mean doses for the liver and small bowel were comparable for all three techniques. The lowest mean doses for the oral cavity, kidneys and lung were found in the FinF technique, while the highest were found in the VMAT technique.

\section{Non-target tissue sparing}

Although the mean doses to non-target tissue were similar for all three techniques (6.27Gy for FinF, 6.87Gy for IMRT and 6.93Gy for VMAT), absolute volume receiving the prescribed dose was significantly lower for the VMAT-CSI. The absolute non-target tissue volumes receiving the prescribed dose were $1031 \mathrm{~cm}^{3}, 1553 \mathrm{~cm}^{3}$ and
$121 \mathrm{~cm}^{3}$ for the FinF, IMRT and VMAT, respectively. Fifty percent of the entire non-target volume (D\%50) was exposed $1.06 \mathrm{~Gy}$ by the FinF, 3.36 Gy by the IMRT and 5.1 Gy by the VMAT.

\section{Dosimetry}

The percentage of dose differences between the calculated doses and the mean of TLD dose readings in each location for the FinF, IMRT and VMAT techniques were the key for evaluating the accuracy of the three-dimensional dose distribution (table 2). In all three techniques, maximum dose differences were found in testicles where located outside the treatment field. The percentage of dose differences in testicles was $9.7 \%, 10.7 \%$ and $12 \%$ for FinF, IMRT and VMAT respectively. Other high dose differences were found in low-density lungs. The percentage of difference was $7.8 \%$ for FinF, $8.2 \%$ for IMRT and $8.4 \%$ for VMAT. Other very important regions to be evaluated were field junctions. The dose differences for those regions were comparable for the three techniques. The highest differences were found in the VMAT technique with $1.6 \%$ in the cervical region and $0.7 \%$ in the lumbar region. The differences between the calculated and measured doses were not found as statistically significant for all three techniques. The $\mathrm{p}$ values were 0.394 , 0.322 and 0.338 for FinF, IMRT and VMAT respectively.

\section{Treatment times and MUs}

The FinF technique provided the minimum monitor units (MU) (511 MU) and the shortest total treatment time $(\sim 22 \mathrm{~min})$. Although the $\mathrm{kV}$ $-\mathrm{kV}$ setup verification time was similar for FinF and IMRT ( $\sim 6 \mathrm{~min})$, it was three times longer for the VMAT-CSI $(\sim 18 \mathrm{~min})$ because of the machine's limitations secondary to the multiple isocenters in one plan. Nevertheless, the total treatment time for the VMAT-CSI was equal to the IMRT technique $(\sim 28 \mathrm{~min})$. The MUs for VMAT and IMRT were $742 \mathrm{MU}$ and $1109 \mathrm{MU}$ respectively. VMAT technique provided a reduction of about 33\% in MU when compared to IMRT. 
Table 2. The percentage of dose differences between the planned and the mean TLD measurements for each technique. \%DD refers to percentage dose difference.

\begin{tabular}{|c|c|c|c|c|c|c|c|c|c|}
\hline \multirow[b]{2}{*}{ Organ } & \multicolumn{3}{|c|}{ VMAT } & \multicolumn{3}{|c|}{ IMRT } & \multicolumn{3}{|c|}{ Finf } \\
\hline & TLD (cGy) & TPS (cGy) & \%DD & TLD (cGy) & TPS (cGy) & $\% \mathrm{DD}$ & TLD (cGy) & TPS (cGy) & \%DD \\
\hline Brain1 & 181 & 187,5 & 3,4 & 190.7 & 188.6 & -1.1 & 187.1 & 188.7 & 0.8 \\
\hline Brain2 & 180,4 & 184,5 & 2,2 & 188.7 & 191.9 & 1.6 & 190.6 & 191.1 & 0.3 \\
\hline Cribriform plate & 30,8 & 30,6 & $-0,8$ & 10.9 & 10.8 & -0.8 & 188.1 & 187.9 & -0.1 \\
\hline Left Eye & 29,4 & 28,1 & $-4,5$ & 15.8 & 15.3 & -3.6 & 18.8 & 19.2 & 1.9 \\
\hline Right Eye & 39,2 & 40,4 & 3 & 17.8 & 16.9 & \begin{tabular}{|l|}
-5.7 \\
\end{tabular} & 18.4 & 19.1 & 3.6 \\
\hline Brainstem & 194,9 & 190 & $-2,6$ & 180.8 & 185.7 & 2.6 & 187.9 & 185.7 & \begin{tabular}{|l|}
-1.2 \\
\end{tabular} \\
\hline Oral Cavity & 197,4 & 190,9 & $-3,4$ & 188.1 & 186.8 & -0.7 & 10.8 & 10.8 & -0.0 \\
\hline Thyroid1 & 57,9 & 57,5 & $-0,4$ & 92.9 & 95.3 & 2.5 & 127.7 & 126.0 & -1.0 \\
\hline Thyroid2 & 33,1 & 33 & $-0,3$ & 47.9 & 46.1 & -3.9 & 104.8 & 108.9 & 3.8 \\
\hline Spinal Cord Cervical (Field Junction) & 173 & 175,9 & 1,6 & 151.7 & 149.7 & -1.3 & 182.7 & 181.3 & -0.7 \\
\hline Lung1 & 27,5 & 30,1 & 8,4 & 23.7 & 25.8 & 8.2 & 4.7 & 5.1 & 7.0 \\
\hline Lung2 & 35,6 & 38,5 & 7,4 & 24.1 & 25.7 & 6.0 & 97.3 & 100.3 & 2.9 \\
\hline Lung3 & 19,1 & 20,6 & 7,3 & 31.9 & 33.8 & 5.7 & 107.0 & 116.1 & 7.8 \\
\hline Lung4 & 39 & 41,6 & 6,8 & 12.5 & 13 & 3.4 & 7.7 & 7.9 & 2.3 \\
\hline Heart1 & 22,2 & 21,2 & $-4,7$ & 12.8 & 12.5 & -2.5 & 7.7 & 8 & 3.4 \\
\hline Heart2 & 43,1 & 45,4 & 5,1 & 32.1 & 32.8 & 2.1 & 5.6 & 5.4 & -4.7 \\
\hline Liver & 73,2 & 71,1 & -3 & 66.7 & 67.3 & 0.8 & 41.9 & 43.8 & 4.1 \\
\hline Right kidney & 33,5 & 31,1 & $-7,7$ & 12.8 & 12.2 & -5.5 & 5.6 & 5.7 & 1.4 \\
\hline Left Kidney & 20 & 19,8 & $-0,9$ & 16.4 & 16.4 & -0.2 & 6.8 & 6.4 & -6.1 \\
\hline Spinal Cord Lumbar1 (FJ) & 181,9 & 183,1 & 0,6 & 184.2 & 185.3 & 0.6 & 191.2 & 190.4 & -0.4 \\
\hline Spinal Cord Lumbar2 (Field Junction) & 184,9 & 186,3 & 0,7 & 198.2 & 199.7 & 0.7 & 155.9 & 155.0 & -0.6 \\
\hline Testicle & 0,4 & 0,4 & -12 & 0.9 & 0.8 & -10.7 & 0.5 & 0.5 & -9.7 \\
\hline
\end{tabular}

\section{DISCUSSION}

Craniospinal irradiation is one of the most difficult and complex treatments in radiation therapy. Both target coverage and sparing of OARs are important for tumor control and toxicities. Treating the spine with a single PA field causes a high exit dose along with all the critical organs, which are placed anteriorly to the spine such as the thyroid, heart and small intestine. Additionally, dose variation in the vertebral body has an effect on asymmetric bone growth in children. The field junctions may produce hot and cold dose areas, which are critical for myelitis and local tumor control failure. For these reasons, clinical advantages and the dosimetric accuracy of new treatment modalities should be investigated and implemented in the routine for CSI. We have evaluated these parameters for the VMAT technique in this study.

A humanlike phantom was planned and irradiated in a supine position for this study. The prone position was preferred in a conventional manner because field junctions can be seen on Int. J. Radiat. Res., Vol. 19 No. 3, July 2021 the patient's skin but overlapping fields are used to provide dose homogeinity by optimizing field fluence for modulated techniques. Additionally, the prone position is not only uncomfortable, but it is also not suitable if anesthesia is required (19-21). An uncomfortable setup can cause intrafractional errors and reduce the dosimetric accuracy of the treatment.

Evaluating the sagittal view with color wash dose distributions shows (figure 3) that the most conformal and homogenous dose distribution, especially in the field junctions, was obtained with the VMAT technique. All three techniques showed good dose coverage for PTV Brain and succeeded to provide more than 95\% of the prescribed dose ( $>34.2 \mathrm{~Gy}$ ) to $95 \%$ of the PTV Brain volume (D95).

The spinal PTV mean, D95 and D2 doses were (36.4, 34.6 and 38.0Gy), (38.8, 36.9 and 40Gy) and (31.9, 31.9 and 39.2Gy) for the VMAT, IMRT and FinF techniques, respectively. Both the VMAT and IMRT plans gave similar results regarding target coverage, but the FinF plan showed insufficient dose coverage, especially in the US region. When D95 doses were evaluated 
in regard to PTVSpinal by the percentage of the prescribed dose, VMAT and IMRT provided more than 95\%, while FinF was able to provide only $88 \%$. These results are significant in evaluating tumor control probability. In addition to poor dose coverage in the spinal region, the highest maximum doses to the heart, thyroid and small bowel were found in the conventional FinF technique. Although the maximum doses for heart, thyroid, lung, liver, intestine and testicle were found to be lower in the VMAT plan than in the other two techniques, the mean doses were found to be higher for all OARs except the heart and thyroid. Brondin NP et al compared 3DCRT, VMAT and intensity modulated proton therapy (IMPT) techniques for CSI and reported that while the risk of developing heart failure, hearing loss, hypothyroidism and xerostomia was highest for the 3D CRT plans, estimates of SC risk was higher for the VMAT plans (4). Studenski et al. compared the DVH results of the same three techniques with our study for 10 adult CSI patients. Although the shortest treatment time was reached with 3DCRT, it also showed the highest maximum OAR doses. Not only did the IMRT have the longest treatment time, but also it had the highest maximum dose in the target. Even so, the IMRT provided the greatest OAR sparing; the VMAT provided the best PTV coverage in the spine region but also delivered the highest mean dose to the OARs. Studenski et al. concluded that although inefficient, the IMRT technique is the most clinically feasible because of the increased mean OAR dose with the VMAT technique, and they warned that providing quality assurance of the beams, especially in the junction regions, is essential (26).

Target, OARs and non-target tissue doses in the VMAT plan of our study were comparable to the results reported in Fogliata et al. (14). They reported the DVH results of the VMAT-CSI treatment plans from five different institutes and compared the results with a Tomotherapy CSI study. The lengths of targets varied between 54.6 and $78.4 \mathrm{~cm}$. While two patients were in a supine position, the other three were in a prone position. Each institute used a different arc arrangement. The mean D95 and D2 doses for
PTV were 34.4 Gy and 38.5 Gy, respectively. The organ at risk mean doses were compared with the Tomotherapy study and reported VMAT vs. Tomotherapy for the heart was 6.7-11 Gy. The mean heart dose was 7.1 Gy in VMAT plan of our study. The mean dose for normal tissue was 5.6 $\mathrm{Gy}$, and the volume receiving the prescription dose (36 Gy) was $37.4 \mathrm{~cm}^{3}$. In conclusion, the VMAT-CSI technique was applied successfully in five different institutes in Europe, and they reported that the VMAT-CSI has some advantages over other techniques. The most important superiority was a shorter beam-on time compared with Tomotherapy $(14,27)$.

Comparing dose distributions on the axial view and DVHs demonstrates that a larger amount of OARs' volumes were exposed to low doses with the IMRT and VMAT as expected. The mean doses for non-target tissue were similar for all three techniques, but the absolute volume receiving the prescribed dose was significantly smaller in the VMAT plan $\left(121 \mathrm{~cm}^{3}\right.$ vs. $1553 \mathrm{~cm}^{3}$ and 1031). Any technique requiring multiple entry angles like VMAT will increase the low-dose bath region that may result in higher secondary malignancy risk especially for children who compose the majority of the patient treated with this technique (22). Galloway et al. reported 16 CSI patients who developed radiation-induced secondary cancers (23). All patients were treated with a conventional technique. The applied dose was categorized into 3 groups: high dose ( $>45 \mathrm{~Gy}$ ), moderate dose (20-36 Gy), and low dose ( $<20$ Gy). The median age of patients who developed second tumors was 7.2 years (range, 2.0-17.4). The most common location of the second tumor was in the whole brain field (57\%) and in the moderate dose range $(81 \%)$. Other sites were the thyroid, spinal region and nasal cavity. Only one patient developed secondary thyroid cancer in the low dose region as a result of exposure to the exit dose of the spinal PA field. In our study, normal tissue volume receiving a 20-36 Gy (moderate) dose range was found significantly lower with the VMAT when compared to the other two techniques. In addition, the mean and maximum dose to the thyroid was found to be significantly lower for the VMAT with respect to 
the FinF. Therefore, one can speculate that this result could be an indicator that a low dose to a larger normal tissue volume with VMAT does not increase the probability of secondary malignancies (24). A more homogeneous dose distribution, better OAR sparing and the same or less probability for an SC might be achieved with proton therapy (25). However, this change is only an advantage if the proton machine employs a pencil-scanning beam. Many proton facilities use passive modulation to produce a field of sufficient size, but the use of a scattering foil produces neutrons, which results in an effective dose to the patient higher than that characteristic of IMRT. The benefit of protons is only achieved if a scanning beam is used in which the doses are 10 times lower than with IMRT (6). However, radiation leakage can be reduced by using VMAT as a result of a decreased total number of MUs than IMRT. There was no assessment of SC risk for treatment techniques that was evaluated in this study.

DVH superiority alone is not enough for transitioning a complex technique such as VMAT -CSI to clinical practice directly. Calculated dose distribution accuracy should be tested in a humanlike phantom for simulating real clinical circumstances as closely as possible. In particular, field junction regions should be investigated carefully as mentioned in other studies that evaluate CSI treatment techniques (12-14,26). Surucu et al. have investigated target and OAR doses for VMAT TMI (Total Marrow Irradiation) and measured calculated-delivered dose differences with TLD. The mean lung dose was $600 \mathrm{cGy}$, and they reported that it is $35 \%$ lower than the pulmonary toxicity cut-off of ' 940 cGy'. In our study, the mean lung dose was limited to 667 cGy, which is $29 \%$ lower than that cut-off. The VMAT TMI is as complex technique as VMAT CSI is because multiple isocenters and overlapping arcs are used in both techniques. TLD measurements were matched with calculated doses, and the median difference was $0.5 \%(-4.3,6.6)$ for VMAT TMI (28). That dose delivery accuracy result can be compared to our results. In our study, median differences between calculated dose and TLD measurements were $0.27 \%(7.8,-9.7)$ for FinF, $0.28 \%(8.1,-10.7)$ for IMRT and $0.33 \%(8.4,-12)$ for VMAT. Although the biggest difference was found for VMAT, the calculated and measured doses were not statistically different for all three techniques. The $\mathrm{p}$ values were 0.394 (FinF), 0.322 (IMRT) and 0.338 (VMAT). The biggest difference was found for VMAT because there are many variables in VMAT. During the gantry rotation, the shapes of multileaf collimators (MLC) are modulated as well as the dose rate, and the gantry rotation speed is optimized. Moreover, a steep dose gradient in the modulated techniques affects the accuracy of point dose measurements. By contrast, gantry and MLC are stationary during irradiation for FinF, and the dose gradient is smoother than with the modulated techniques. Besides the testicle, the maximum dose differences were found in the lungs for all techniques because of the non-homogeneous medium. It is known that AAA calculates the dose higher than realty for a low-intensity medium like the lung $(29,30)$. The testicle is located in the peripheral region, and it is exposed to very low doses between 0.15 and 0.25 Gy. Therefore, small differences between the calculated and measured doses cause a big percentage difference. Additionally, field junctions are the most important areas where the compliance between the planned and irradiated dose should be investigated. For the VMAT-CSI, calculated and measured doses showed good agreement both in the upper and lower field junctions. The differences were $1.6 \%$ and $0.7 \%$, respectively. To increase the accuracy of the calculation, algorithms that can calculate dose distribution as accurately as Monte Carlo can be chosen $(31,32,33)$.

In addition to evaluating DVH parameters and dosimetric accuracy of treatment techniques, MU values and total treatment times should also be evaluated because reducing these parameters is important to reduce radiation leakage and intrafractional errors. The FinF provided the most efficient delivery with the minimum of MUs, the shortest beam on, and the shortest treatment time. Although the total treatment times, including setup verifications, were comparable for IMRT and VMAT, the actual 
MUs and beam on time were shorter for VMAT than for IMRT. Comparison of MUs between VMAT and IMRT was compatible with the literature; VMAT technique provided a reduction of about 33\% in MU when compared to IMRT (34).

\section{CONCLUSION}

VMAT-CSI was found to be the superior technique in terms of delivering a prescription dose to the target structures and sparing normal tissues from higher doses. However, the risk of secondary cancer based on increased low dose volume should be evaluated for individual cases. The encouraging dosimetric results in regard to the TLD measurements in a humanlike phantom corroborate that the VMAT-CSI technique can be used clinically as confidently as conventional FinF and IMRT techniques.

\section{ACKNOWLEDGMENT}

This study was done at Istanbul University, Oncology Institute and was supported by Yildiz Technical University Scientific Research project coordinatorship (2012-01-01-DOP05).

Presentations: This paper was presented at the AAPM 2014 Innovation, 56th Annual Meeting \& Exhibition, Texas, United States of America.

\section{Conflicts of interest: Declared none.}

\section{REFERENCES}

1. Mehta MP, Chang S M, Guha A, Newton HB, Vogelbaum MA (2011) Principles \& practice of neuro-oncology: A multidisciplinary approach. New York, Demos Medical.

2. Olch AJ (2013) Pediatric radiotherapy treatment and planning. Florida, Taylor \& Francis Group.

3. Chang EL, Wong PF, Forster KM, Petru MD, Kowalski AV, Maor MH (2003) Verification techniques and dose distribution for computed tomographic planned supine craniospinal radiation therapy. Med Dos, 28(2): 127-131.

4. Brodin NP, AF Rosenschöld PM, Aznar MC, Kiil-Berthelsen
A, Vogelius IR, Nilsson P, Lannering B, Björk-Eriksson T (2011) Radiobiological risk estimates of adverse event and secondary cancer for proton and photon radiation therapy of pediatric medulloblastoma. Acta Oncologica, 50: 806816.

5. Yoon M, Shin DH, Kim J, Kim JW, Kim DW, Park SY, Lee SB, Kim YJ, Park HJ, Park BK, Shin SH (2011) Craniospinal irradiation techniques: a dosimetric comparison of proton beams with standard and advanced photon radiotherapy. Int J Radiat Oncol Biol Phys, 81: 637-46.

6. Hall EJ (2006) Intensity-Modulated Radiation Therapy, Protons, and the Risk of Second Cancers. Int J Radiat Oncol Biol Phys, 65(1): 1-7.

7. Zhang $P$, Happersett L, Hunt M, Jackson A, Zelefsky M, Mageras G (2010) Volumetric modulated arc therapy: planning and evaluation for prostate cancer cases. Int $J$ Radiat Oncol Biol Phy, 76: 1456-1462.

8. Otto K (2008) Volumetric modulated arc therapy: IMRT in a single gantry arc. Med Phys, 35: 310-317.

9. Aydogan B, Yeginer M, Kavak GO, Fan J, Radosevich JA, Gwe-Ya K (2011) Total marrow irradiation with RapidArc volumetric arc therapy. Int I Radiat Oncol Biol Phys, 81: 592-599.

10. Seravalli E, Bosman M, Lassen-Ramshad $Y$, Vestergaard A, Oldenburger F, Visser J, Koutsouveli E, et al. (2018) Dosimetric comparison of five different techniques for craniospinal irradiation across 15 European centers: analysis on behalf of the SIOP-E-BTG (radiotherapy working group). Acta Oncologica, 57(9):1240-1249.

11. Fogliata A, Bergström S, Cafaro I, Clivio A, Cozzi L, Dipasquale $G$, Hållström $P$, Mancosu $P$, Navarria $P$, Nicolini G, Parietti E, Pesce GA, Richetti A, Scorsetti M, Vanetti E, Weber DC (2007) Cranio-spinal irradiation with volumetric modulated Arc therapy: A multi-institutional treatment experience. Radiother Oncol, 99: 79-85.

12. Baghani HR, Aghamiri SMR, Gharaati H, Mahdavi SR, Hosseini Daghigh SM (2011) Comparing the results of 3D treatment planning and practical dosimetry in craniospinal radiotherapy using Rando phantom. Int J Radiat Res, 9(3): 151-158.

13. Hood C, Kron T, Hamilton C, Callan S, Howlett S, Alvaro F, Back M (2005) Correlation of 3D-planned and measured dosimetry of photon and electron craniospinal radiation in a pediatric anthropomorphic phantom. Radiotherapy and Oncology, 77(1): 111-6.

14. Shoa P, Abedi I, Tavakoli MB, Amouheidari AR, Jabbari $K$ (2020) Estimation of the visual system complication probability on children with Medulloblastoma after Craniospinal irradiation with three-dimensional conformal radiotherapy. Int J Radiat Res, 18(1): 117-23.

15. Pai Panandiker A, Ning H, Likhacheva A, Ullman K, Arora B, Ondos J, Karimpour S, Packer R, Miller R, Citrin D (2007) Craniospinal irradiation with spinal IMRT to improve target homogeneity. Int J Radiat Oncol Biol Phys, 68(5): 1402 $-1409$.

16. Kusters JMAM, Louwe RJW, Van Kollenburg PGM, KunzeBusch MC, Gidding CEM, Van Lindert EJ, Kaanders JHAM, Janssens GORJ (2011) Optimal Normal Tissue Sparing In

Int. J. Radiat. Res., Vol. 19 No. 3, July 2021 
Craniospinal Axis Irradiation Using IMRT With Daily Intrafractionally Modulated Junction(S). Int J Radiat Oncol Biol Phys, 81(5): 1405-1414.

17. Parker W, Filion E, Roberge D, Freeman CR (2007) Intensity-modulated radiotherapy for craniospinal irradiation: Target volume considerations, dose constraints and competing risks. Int J Radiat Oncol Biol Phys, 69(1): 251-257.

18. Wang K, Meng H, Chen J, Zhang W, Feng Y (2018) Plan quality and robustness in field junction region for craniospinal irradiation with VMAT. Phys Med, (48): 21-26.

19. South M, Chiu JK, Teh BS, Bloch C, Schroeder TM, Arnold CP (2008) Supine craniospinal irradiation using intrafractional junction shifts and field-in-field dose shaping: Early experience at methodist hospital. Int J Radiat Oncol Biol Phys, 71(2): 477-483.

20. Hideghéty K, Cserháti A, Nagy Z, Varga Z, Fodor E, Vincze V, Szántó E, Maráz A, Thurzó LA (2012) Prospective Study of Supine versus Prone Positioning and Whole-Body Thermoplastic Mask Fixation for Craniospinal Radiotherapy in Adult Patients. Radiother Oncol, 102(2): 214-218.

21. Verma J, Mazloom A, Teh SB, South M, Butler EB (2015) Comparison of supine and prone craniospinal irradiation in children with medulloblastoma. Pract Radiat Oncol, 5(2): 93-98.

22. Athiyamana $H$, Mayilvaganana $A$, Chouguleb $A$, Joanb $M$, Singh Kumarc $H$ (2019) Estimation of radiation-induced second cancer risk associated with the institutional field matching craniospinal irradiation technique: A comparative treatment planning study. Reports of Practical Oncology and Radiotherapy, 24: 409-420.

23. Galloway TJ, Indelicato DJ, Amdur RJ, Morris CG, Swanson EL, Marcus RB (2012) Analysis of Dose at the Site of Second Tumor Formation after Radiotherapy to the Central Nervous System. Int J Radiat Oncol Biol Phys, 82(1): 90-94.

24. Neglia JP, Friedman DL, Yasui $Y$, Mertens AC, Hammond $S$, Stovall M, Donaldson SS, Meadows AT, Robison LL (2001) Second Malignant Neoplasms in Five-Year Survivors of Childhood Cancer: Childhood Cancer Survivor Study. J Natl Cancer Inst, 93(8): 618-629.

25. St. Clair WH, Adams JA, Bues M, Fullerton BC, La Shell S, Kooy HM, Loeffler JS, Tarbell NJ (2004) Advantage of Protons Compared to Conventional X-Ray or IMRT in the
Treatment of a Pediatric Patient with Medulloblastoma. Int J Radiat Oncol Biol Phys, 58(3): 727-734.

26. Studenski MT, Shen X, Yu Y, Xiao Y, Shi W, Biswas T, Werner-Wasik M, Harrison AS (2013) Intensity-Modulated Radiation Therapy and Volumetric-Modulated Arc Therapy for Adult Craniospinal Irradiation-A Comparison with Traditional Techniques. Med Dos, 38(1): 48-54.

27. Parker W, Brodeur M, Roberge D, Freeman C (2010) Standard and nonstandard craniospinal radiotherapy using helical TomoTherapy. Int J Radiat Oncol Biol Phys, 77: 926 -931 .

28. Surucu M, Yeginer M, Kavak GO, Fan J, Aydogan B (2012) Verification of Dose Distribution for Volumetric Modulated Arc Therapy Total Marrow Irradiation in a Humanlike Phantom. Med Phys, 39(1): 281-288.

29. Bragg CM and Conway J (2006) Dosimetric Verification of the Anisotropic Analytical Algorithm for Radiotherapy Treatment Planning. Radiother Oncol, 81(3):315-332.

30. Van Esch A, Tillikainen L, Pyykkonen J, Tenhunen M, Helminen $\mathrm{H}$, Siljamäki $\mathrm{S}$, Alakuijala J, Paiusco $\mathrm{M}$, lori $\mathrm{M}$, Huyskens DP (2006) Testing of the Analytical Anisotropic Algorithm for Photon Dose Calculation. Med Phys, 33(11): 4130-4148.

31. Han T, Mourtada F, Kisling K, Mikell J, Followill D, Howell R (2012) Experimental Validation of Deterministic Acuros XB Algorithm for IMRT and VMAT Dose Calculations with the Radiological Physics Center's Head and Neck Phantom. Med Phys, 39(4): 2193-2202.

32. Kan MWK, Leung LHT, So WKR, Yu PKN (2013) Experimental Verification of the Acuros XB and AAA Dose Calculation Adjacent to Heterogeneous Media for IMRT and RapidArc of Nasopharygeal Carcinoma. Med Phys, 40(3): 031714-1-19.

33. Kim YL, Suh TS, Choe BY, Choi BO, Chung JB, Lee JW, Bae YK, Park BM, Jung JY, Shin YJ, Kim JY, Moon SK (2016) Dose distribution evaluation of various dose calculation algorithms in inhomogeneous media. Int I Radiat Res, 14(4): 269-78.

34. Hansen AT, Lukacova S, Ramshad YL, Petersen JB (2015) Comparison of a new noncoplanar intensity-modulated radiation therapy technique for craniospinal irradiation with 3 coplanar techniques. Med Dos, 40: 296-303. 
